\title{
TEORÍA DE LA EDUCACIÓN. REVISTA INTERUNIVERSITARIA
}

\section{ISSN 1130-3743}

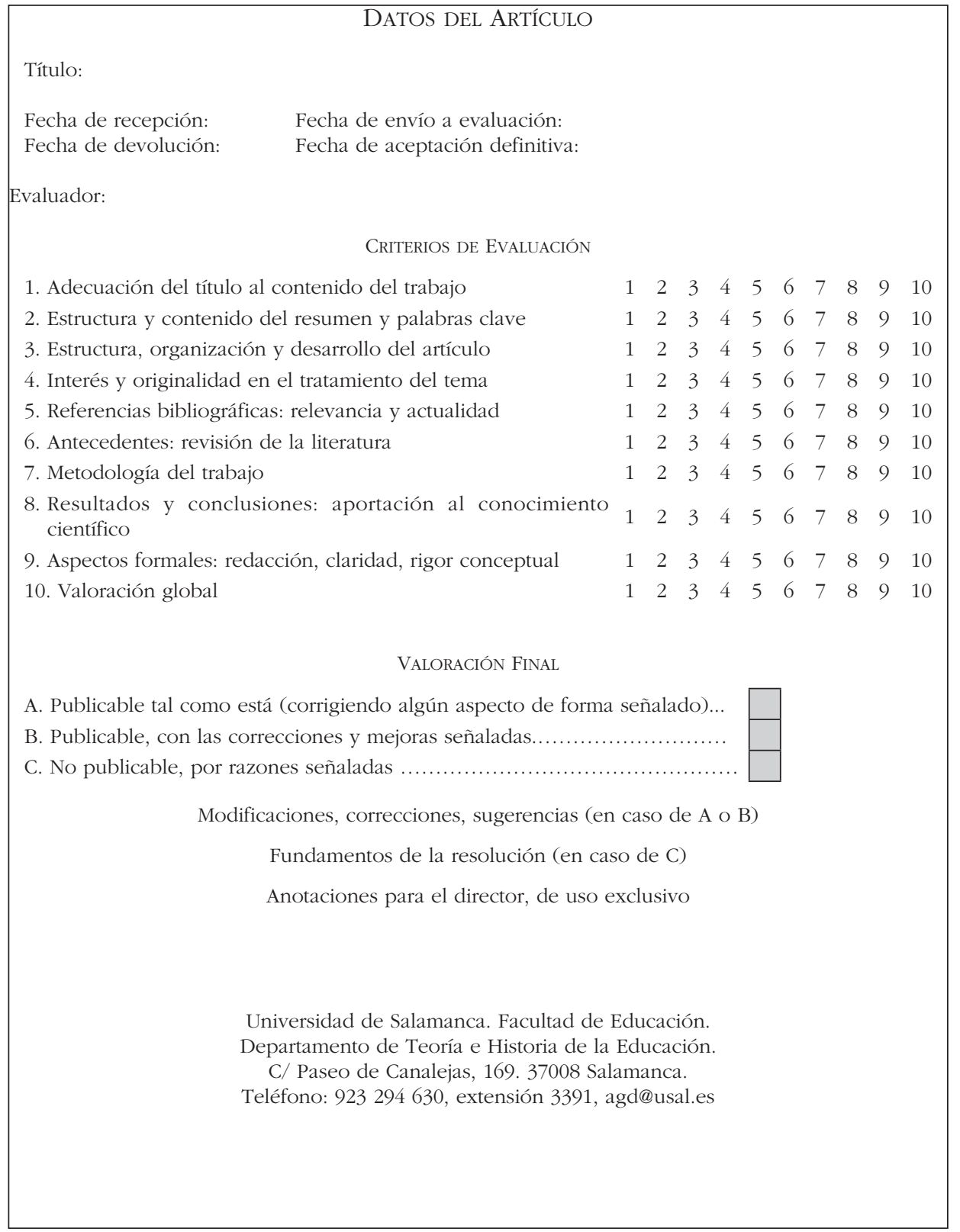




\section{TEORÍA DE LA EDUCACIÓN. REVISTA INTERUNIVERSITARIA}

ISSN 1130-3743

\section{ARTICLE INFORMATION}

Title:

Date of reception:

Date returned:
Date sent for review:

Date of final acceptance:

Reviewer:

\section{ASSESSMENT CRITERIA}

1. The title is in line with the content of the work

2. Structure and contents of abstract and key words

3. Structure, organization and development of the article

4. Interest of and originality in the treatment of the topic

5. Bibliographical references: relevance and topicality

6. Background: literature review

7. Work methodology

8. Results and conclusion: contribution to scientific knowledge

9. Formal aspects: writing, clarity, conceptual rigour

10. Overall assessment

$\begin{array}{llllllllll}1 & 2 & 3 & 4 & 5 & 6 & 7 & 8 & 9 & 10 \\ 1 & 2 & 3 & 4 & 5 & 6 & 7 & 8 & 9 & 10 \\ 1 & 2 & 3 & 4 & 5 & 6 & 7 & 8 & 9 & 10 \\ 1 & 2 & 3 & 4 & 5 & 6 & 7 & 8 & 9 & 10 \\ 1 & 2 & 3 & 4 & 5 & 6 & 7 & 8 & 9 & 10 \\ 1 & 2 & 3 & 4 & 5 & 6 & 7 & 8 & 9 & 10 \\ 1 & 2 & 3 & 4 & 5 & 6 & 7 & 8 & 9 & 10 \\ 1 & 2 & 3 & 4 & 5 & 6 & 7 & 8 & 9 & 10 \\ 1 & 2 & 3 & 4 & 5 & 6 & 7 & 8 & 9 & 10 \\ 1 & 2 & 3 & 4 & 5 & 6 & 7 & 8 & 9 & 10\end{array}$

FINAL ASSESSMENT

A. Publishable as is (with correction of formal aspects indicated).

B. Publishable, with the corrections and improvements indicated

C. Not publishable.

Modifications, corrections, suggestions (If A B)

Basis of decision (If C)

Notes exclusively for the editor

Universidad de Salamanca. Facultad de Educación.

Departamento de Teoría e Historia de la Educación.

C/ Paseo de Canalejas, 169. 37008 Salamanca.

Telephone: 923294 630, extension 3391, agd@usal.es 


\section{TEORÍA DE LA EDUCACIÓN. REVISTA INTERUNIVERSITARIA}

ISSN 1130-3743

\section{DONNÉES DE L'ARTICLE}

Titre:

Date de réception:

Date de renvoi:

Referee:

\section{CRITÈres D'ÉVALUATION}

1. Adéquation du titre aux contenus du travail

$\begin{array}{llllllllll}1 & 2 & 3 & 4 & 5 & 6 & 7 & 8 & 9 & 10\end{array}$

2. Structure et contenu du résumé et mots clés

$\begin{array}{llllllllll}1 & 2 & 3 & 4 & 5 & 6 & 7 & 8 & 9 & 10\end{array}$

3. Structure, organisation et développement de l'article

4. Intérêt et originalité du sujet

5. Références bibliographiques: importance et nouveauté

6. Précédents: révision de la littérature

7. Méthodologie du travail

$\begin{array}{llllllllll}1 & 2 & 3 & 4 & 5 & 6 & 7 & 8 & 9 & 10\end{array}$

$\begin{array}{llllllllll}1 & 2 & 3 & 4 & 5 & 6 & 7 & 8 & 9 & 10\end{array}$

$\begin{array}{llllllllll}1 & 2 & 3 & 4 & 5 & 6 & 7 & 8 & 9 & 10\end{array}$

$\begin{array}{llllllllll}1 & 2 & 3 & 4 & 5 & 6 & 7 & 8 & 9 & 10\end{array}$

$\begin{array}{llllllllll}1 & 2 & 3 & 4 & 5 & 6 & 7 & 8 & 9 & 10\end{array}$

8. Résultats et conclusions: apports à la connaissance scientifique

$\begin{array}{llllllllll}1 & 2 & 3 & 4 & 5 & 6 & 7 & 8 & 9 & 10\end{array}$

$\begin{array}{llllllllll}1 & 2 & 3 & 4 & 5 & 6 & 7 & 8 & 9 & 10\end{array}$

$\begin{array}{llllllllll}1 & 2 & 3 & 4 & 5 & 6 & 7 & 8 & 9 & 10\end{array}$

10. Qualification globale

\section{QuALIFICATION GLOBALE}

A. Publiable tel qu'il est (en corrigeant quelques aspects formels signalés).....

B. Publiable avec les corrections et améliorations signalées

C. Non publiable

Modifications, corrections, suggestions (si A ou B)

Base de la décision (C)

Observations pour le directeur, d'utilisation exclusive

Universidad de Salamanca. Facultad de Educación.

Departamento de Teoría e Historia de la Educación.

C/ Paseo de Canalejas, 169. 37008 Salamanca.

Telephone: 923294 630, extension 3391, agd@usal.es 